\title{
Cooperative Adaptive Cruise Control Using Delay-Based Spacing Policy: A Robust Adaptive Non-Singular Terminal Sliding Mode Approach
}

\author{
$W A N G$ Weiyang ${ }^{1,2}$ (王维旸), $C U I K e^{1 *}$ (崔 科), GU Lizhong ${ }^{1}$ (顾立忠), $L \ddot{U} X_{\text {injun }}^{1}$ (吕新军) \\ (1. CASCO Signal Ltd., Shanghai 200072, China;
}

2. Department of Automation, Shanghai Jiao Tong University, Shanghai 200240, China)

(C) The Author(s) 2021

\begin{abstract}
This study proposes two speed controllers based on a robust adaptive non-singular terminal sliding mode control approach for the cooperative adaptive cruise control problem in a connected and automated vehicular platoon. The delay-based spacing policy is adopted to guarantee that all vehicles in the platoon track the same target velocity profile at the same position while maintaining a predefined time gap. Factors such as nonlinear vehicle longitudinal dynamics, engine dynamics with time delay, undulating road profiles, parameter uncertainties, and external disturbances are considered in the system modeling and controller design. Different control objectives are assigned to the leading and following vehicles. Then, controllers consisting of a sliding mode controller with parameter adaptive laws based on the ego vehicle's state deviation and linear coupled state errors, and a Smith predictor for time delay compensation are designed. Both inner stability and strong string stability are guaranteed in the case of nonlinear sliding manifolds. Finally, the effectiveness of the proposed controllers and the benefits of $44.73 \%$ shorter stabilization time, $11.20 \%$ less speed overshoot, and virtually zero steady-state inner vehicle distance deviation are illustrated in a simulation study of a seven-vehicle platoon cooperative adaptive cruise control and comparison experiments with a coupled sliding mode control approach.
\end{abstract}

Key words: cooperative adaptive cruise control, delay-based spacing policy, adaptive non-singular terminal sliding control, string stability

CLC number: U 495

Document code: A

\section{Introduction}

Significant developments in connected and automated vehicle $(\mathrm{CAV})$ technology have been realized during the last decade. Intelligent CAVs integrated by connectivity and automation can not only drive by themselves with on-board computers and sensing sensors, but also communicate with each other through vehicle-to-vehicle (V2V) communications. Cooperative adaptive cruise control (CACC) is one of the most meaningful technologies for CAVs and has complemented adaptive cruise control (ACC) with cooperative maneuvers. In CACC systems, CAVs share their own parameters and real-time state information (e.g., acceleration, speed, and position) with other CAVs in the network through V2V communications.

Using CACC systems, potential benefits can be achieved, such as increasing traffic capacity by reducing

Received: 2020-11-30 Accepted: 2021-01-04

Foundation item: the Research Project of CASCO Signal Ltd. (No. RE.Z0120032)

*E-mail: cuike@casco.com.cn the gaps between vehicles, reducing energy consumption due to unnecessary speed changes, and reducing aerodynamic drag on the following vehicles, which can achieve up to $10 \%$ energy conservation ${ }^{[1]}$ and improve driving safety ${ }^{[2-3]}$.

In order to ensure the safe CACC operation of such platoons with shorter vehicle-following gaps, cooperative automation of vehicle longitudinal dynamics is required. The two fundamental aspects of the resulting behavior are the spacing policy and cooperative control approach.

Spacing policy, which specifies the desired (and not necessarily static) inter-vehicular distance, is mainly categorized into three policy types: constant spacing $(\mathrm{CS})^{[4]}$, constant time-headway $(\mathrm{CTH})^{[5]}$, and delaybased (D-B) ${ }^{[6]}$.

Different control methods have been adopted in cooperative controller design, including yet not limited to linear feedback control ${ }^{[7-9]}$, optimal control $(\mathrm{OC})$ based approach ${ }^{[10]}$, sliding mode control (SMC) ${ }^{[11-15]}$, and other methods such as model predictive control $(\mathrm{MPC})^{[16-17]}, H_{\infty}$ control $^{[18-20]}$, intelligent $\operatorname{method}^{[21]}$, 
and hybrid methods such as integrated OC with $\mathrm{SMC}^{[22]}$, MPC with $\mathrm{SMC}^{[23]}$, and neural networks (NNs) with $\mathrm{SMC}^{[24]}$.

Among the above controller design approaches, SMC, a well-known nonlinear method with robustness against bounded matched uncertainties, has attracted considerable attention and has been adopted by different researchers to solve the CACC problem. Kwon and Chwa ${ }^{[11]}$ proposed a coupled SMC (CSMC) to improve the performance and string stability of bidirectional platoon control. The coupled sliding surfaces, which are in the form of a linear combination of the ego vehicle's sliding surface and its adjacent vehicles, are designed to guarantee string stability. $\mathrm{Wu}$ et al. ${ }^{[12]}$ proposed a distributed SMC method for homogeneous vehicular platoons based on nonlinear dynamics and generic topologies whose associated matrices are positive definite. The distributed SMC design is divided into two parts: topological sliding surface and topological reaching law designs. Considering uncertain and time-varying driving resistance, Yan et al. ${ }^{[13]}$ proposed a distributed adaptive SMC for platoon control. Then, an adaptive control method was applied to estimate the uncertain resistance coefficients. A coupled sliding surface, which is adopted to link two standalone sliding surface of a single vehicle, ensures satisfactory convergence and string stability.

The above studies are based on bi-level sliding surfaces, which are a lower-level sliding surface constructed by a linear combination of ego-vehicle state errors and an upper level sliding surface constructed by the linear coupled lower sliding surfaces.

Based on the transformed error, two adaptive SMC schemes based on leader-predecessor and leaderbidirectional information flows were presented by Guo and $\mathrm{Li}^{[14]}$ to ensure string stability and strong string stability, respectively. A new linear sliding surface with the proposed transformed error, which is forced into the allowable region, was adopted. Factors such as actuator saturation nonlinearity, which is approximated by a smooth hyperbolic tangent function, uncertain parameters, and unknown disturbances, are also considered. A terminal SMC such as a switching nonlinear sliding manifold was designed in Ref. [15]. The string stability is guaranteed by using the proposed novel spacing error of the vehicles when the sliding surface is reached in finite time.

Different approaches have been combined with SMC methods. Guo and $\mathrm{Li}^{[22]}$ integrated Pontryagin's minimum principle-based OC method with SMC for CACC set-point optimization and tracking control. A twolayered control architecture consisting of a fuel-timeefficient speed-planning algorithm and distributed SMC speed tracking is presented. Fesharaki et al. ${ }^{[23]}$ integrated MPC with SMC for constrained nonlinear systems. A tractable nonlinear MPC is designed based on the nominal system, and the SMC provides the robustness and recovery of the performance against modeling error and system uncertainties. Guo et al. ${ }^{[24]}$ proposed a Chebyshev NN (CNN)-based distributive integral SMC with special consideration for input saturation. CNNs are used to approximate unknown nonlinear functions in the followers online.

However, the majority of the above-mentioned works use a time domain-based spacing policy such as CS or CTH. Only limited studies ${ }^{[6,10]}$ are based on D-B policy. Motived by the fact that the slopes, curvatures, speed limits, and other infrastructure information are all position-based, rather than time domain, a D-B space policy that ensures the same velocity and acceleration in the same location for each vehicle is more desirable and reasonable to be chosen as the target profile. In what follows, two scenarios where the CS or CTH policies are adopted are chosen as an example, explaining the reason for selecting the D-B policy. For a platoon of heavy-duty vehicles traversing a hilly road segment, owing to the length of the platoon, the leading vehicle could accelerate in level or downhill front segments while the following vehicles pass through the steep uphill segments. Not all of the following vehicles can necessarily achieve speed acceleration owing to the road geometry and limitation of their engine power, although the two spacing policies command all vehicles to maneuver synchronously. This scenario was recognized in experiments of Ref. [1]. A lower speed limit can frequently exist in curvature segments, ensuring safety issues. However, the leading vehicle could accelerate after passing through the curvature segment, forcing the following vehicles to accelerate because of the two policies, even though they remain in the curvature segments.

Furthermore, vehicle nonlinear dynamics, parameter uncertainties, external disturbances, and pure delay processes caused by communication delays and engine actor lags are seldom considered simultaneously. To resolve the above issue in $\mathrm{CACC}$ with $\mathrm{D}-\mathrm{B}$ policy, non-singular terminal SMC (NTSMC), which was first proposed by Feng et al. ${ }^{[25]}$ with features that ensure states of the resulting closed-loop system converge to the equilibrium point in finite time while avoiding the singularity problem in conventional terminal SMC, is adopted in this study.

In this paper, two controllers, for the leading vehicle and following vehicles, are proposed for the different control objectives. Factors such as the response process with engine time delay, nonlinearities of the vehicle dynamics, parameter uncertainties, and road vertical profiles are all considered in the CACC controller design.

The remainder of this paper is organized as follows: Section 1 describes a space domain-based CACC problem and the longitudinal dynamics of vehicles in the 
platoon. Section 2 provides a detailed design of the CACC controllers, including system structure, control laws, parameter adaption laws, and proofs of the inner and string stability. Section 3 provides numerical simulations and Section 4 compares the proposed method with the CSMC approach. Section 5 concludes the paper.

\section{Problem Description and Vehicle Model}

\subsection{Space Domain-Based CACC Problem and Delay-Based Spacing Policy}

Consider a vehicular platoon consisting of $n$ heterogeneous CAVs that traverse on a horizontal road on an undulate vertical segment profile with a predefined distance-based speed profile. The predecessorleader following the information flow topology with the optional last-to-ego vehicle communication link is adopted, as displayed in Fig. 1.

Categorized by the different roles in the vehicular platoon, different control objectives are adopted in the driving of a vehicle.

(1) For the leading vehicle, the objective is to track the predefined speed profile computed based on the ego position of the current time as the independent variable:

$$
\left.\begin{array}{l}
x_{2,1}\left(x_{1,1}\right) \rightarrow x_{\mathrm{r} 2,1}\left(x_{1,1}\right) \\
\dot{x}_{2,1}\left(x_{1,1}\right) \rightarrow \dot{x}_{\mathrm{r} 2,1}\left(x_{1,1}\right)
\end{array}\right\},
$$

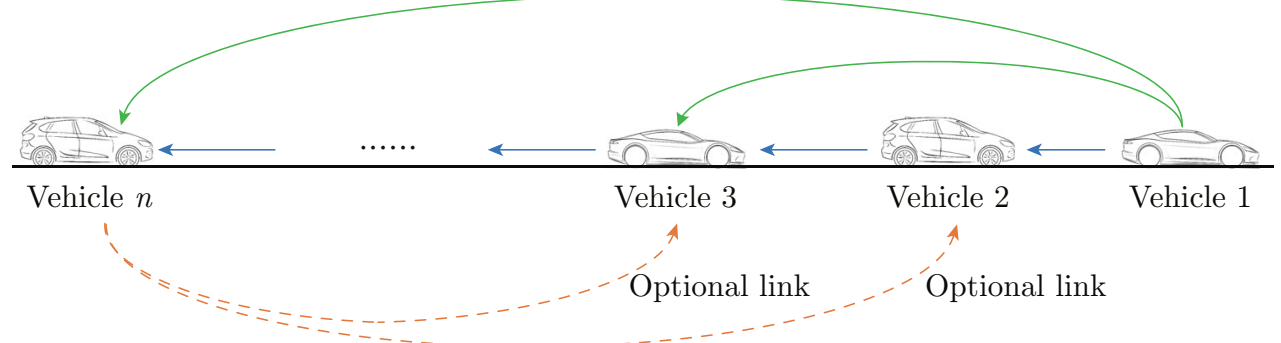

Fig. 1 Information topology of CACC platoon with predecessor-leader following and optional last-to-ego vehicle link

where $x_{1,1}$ and $x_{2,1}$ are the position and velocity of the leading vehicle, respectively, $\dot{x}_{2,1}$ is the time derivative of the leading vehicle's velocity (acceleration), and $x_{\mathrm{r} 2,1}$ and $\dot{x}_{\mathrm{r} 2,1}$ are the target speed and acceleration, respectively. Without loss of generality, " $\rightarrow$ " denotes the tracking process.

(2) For the following vehicles, the objective is to track the D-B position and speed profile obtained from the preceding or leading vehicle, while considering the distance gap between the preceding vehicle, leading vehicle, and optional last vehicle. Thus, the target speed profile for the $i$ th following vehicle $x_{\mathrm{r} 2, i}$ in the D-B spacing policy is given as

$$
\begin{aligned}
& x_{\mathrm{r} 2, i}(t)=x_{\mathrm{r} 2, i-1}\left(t-t_{g, i-1}\right) \\
& \text { or } \\
& x_{\mathrm{r} 2, i}(t)=x_{\mathrm{r} 2,1}\left(t-\sum_{j=1}^{i-1} t_{g, j}\right) \\
& \quad i=2,3, \cdots, n,
\end{aligned}
$$

where $t_{g, i-1}>0$ is the time gap between the $i$ th vehicle and its preceding vehicle.

(3) Stability performance: controllers for the leading and following vehicles must fulfill the inner stability; controllers for the following vehicles must fulfill the string stability.

\subsection{Longitudinal Dynamics of a Vehicle}

The longitudinal dynamics of the $i$ th vehicle in a platoon can be obtained by Newton's second law as follows:

$$
\left.\begin{array}{l}
\dot{x}_{1, i}=x_{2, i} \\
m_{i} \dot{x}_{2, i}=u_{\mathrm{e}, i}-f_{\mathrm{R}, i}+d_{i}
\end{array}\right\},
$$

where $x_{1, i}$ and $x_{2, i}$ are the position and velocity of the vehicle, $m_{i}$ is the static mass of the vehicle, $u_{\mathrm{e}, i}$ is the actual driving force that the engine outputs, $d_{i}$ is the unknown external disturbances such as model errors, uncertainties of the vehicle, road profiles and unpredicted wind gusts, and $f_{\mathrm{R}, i}$ is the total resistance of the vehicle which is attributed to the basic resistance $f_{\mathrm{b}, i}$ and road vertical profile-based resistance $f_{\mathrm{g}, i}$. The basic resistance $f_{\mathrm{b}, i}$ is a consequence of the rolling and speed-related aerodynamic resistances. The road vertical profile-based resistance $f_{\mathrm{g}, i}$ is a consequence of the road slope $\alpha\left(x_{1, i}\right)$. In Eq. (4), $c_{0, i}=r_{i} g$ where $r_{i}$ is the rolling resistance coefficient, $c_{1, i}=0$, and $c_{2, i}=h_{\mathrm{a}, i} A_{i} C_{\mathrm{d}, i} /\left(2 m_{i}\right)$ where $h_{\mathrm{a}, i}$ is the air density, $A_{i}$ is the vehicle front area, $C_{\mathrm{d}, i}$ is the air drag coefficient, and $g$ is the acceleration due to gravity. Typically, the 
grade of the slope on the road is small, and approximations of $\alpha \approx \tan \alpha \approx \sin \alpha$ and $\cos \alpha \approx 1$ are used above. In this study, we assume that the unknown disturbance satisfies $\left|d_{i}\right| \leqslant \bar{d}_{i}$, where $\bar{d}_{i}$ is the upper bound of the disturbance.

Owing to the system inertia, mechanical properties, and transmission delay, the engine dynamics are modeled as a first-order plus dead time (FOPDT) process:

$$
\dot{u}_{\mathrm{e}, i}(t)=-\frac{1}{T_{i}} u_{\mathrm{e}, i}(t)+\frac{1}{T_{i}} u_{\mathrm{c}, i}\left(t-\tau_{i}\right),
$$

where $\dot{u}_{\mathrm{e}, i}$ is the time derivative of $u_{\mathrm{e}, i}, u_{\mathrm{c}, i}$ is the control command input such as the throttle/brake command, $T_{i}$ is the engine time constant, and $\tau_{i}$ is the pure delay time (including the wireless communication network owing to the queuing, contention, transmission, and propagation) for the control command.

\section{CACC Controller Design}

\subsection{Controller Structure}

The proposed controller is composed of two main parts: the adaptive NTSMC-based trajectory tracking controller and Smith predictor (SP), as indicated in Fig. 2. Based on the different roles of the vehicles, different data are chosen as the target input, such as target speed and acceleration based on the position of the leading vehicle, and D-B target and state profile of the leading, preceding, and last vehicles. In every control cycle, the control command computed by the adaptive NTSMC is sent to the simulation (or real) vehicle, as well as the two nominal models with and without the engine delay process. Then, a modified state, $\boldsymbol{x}_{\mathrm{wo}}+\left(\boldsymbol{x}-\boldsymbol{x}_{\mathrm{wd}}\right)$, is sent back to the adaptive NTSMC controller, where $\boldsymbol{x}_{\text {wo }}$ is the vehicle state computed by the nominal model without delay, $\boldsymbol{x}_{\mathrm{wd}}$ is the vehicle state from the nominal model with delay, and $\boldsymbol{x}$ is the real vehicle state. If the nominal model is the same as the real model, the correct term $\boldsymbol{x}-\boldsymbol{x}_{\mathrm{wd}}$ equals zero and the pure delay process has no influence on the controller design.

Thus, the pure delay effects in the engine dynamics

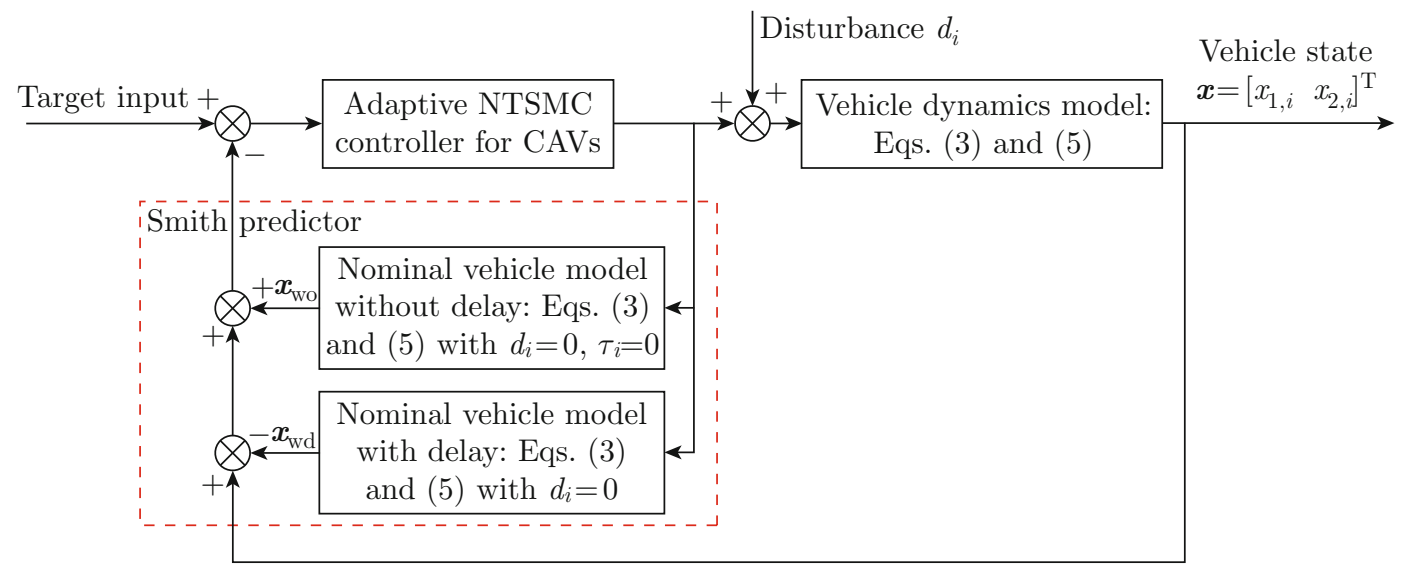

Fig. 2 Framework of proposed CACC controller for CAVs

model are ignored in the following adaptive NTSMC controller design: Consider a third-order vehicle model with engine dynamics based on Eqs. (3) and (5) with $\tau_{i}=0$, define the auxiliary vectors $\boldsymbol{\theta}_{i}=\left(m_{i} c_{0, i}+\right.$ $\left.f_{\mathrm{g}, i}, m_{i} c_{1, i}, m_{i} c_{2, i}\right)$ and $\boldsymbol{\zeta}_{i}=\left(1, x_{2, i}, x_{2, i}^{2}\right)$; then, the total resistance of the vehicle can be rewritten as $f_{\mathrm{R}, i}=\boldsymbol{\theta}_{i} \boldsymbol{\zeta}_{i}^{\mathrm{T}}(x)$. With unknown disturbance terms $d_{i}$ neglected, the vehicle dynamic model for the adaptive NTSMC design is then described as

$$
\left.\begin{array}{l}
\dot{x}_{1, i}=x_{2, i} \\
\dot{x}_{2, i}=\frac{u_{\mathrm{e}, i}-\boldsymbol{\theta}_{i} \dot{\boldsymbol{\zeta}}_{i}^{\mathrm{T}}(x)}{m_{i}}
\end{array}\right\},
$$

and

$$
\dot{u}_{\mathrm{e}, i}=-\frac{1}{T_{i}} u_{\mathrm{e}, i}+\frac{1}{T_{i}} u_{\mathrm{c}, i} .
$$

\subsection{Cruise Controller for Leading Vehicle}

\subsubsection{Control Law Design for Leading Vehicle}

For the leading vehicle $(i=1)$, based on the three-order model in Eqs. (6) and (7) with a predefined distance-based reference speed profile $x_{\mathrm{r} 2, i}$ and its derivative $\dot{x}_{\mathrm{r} 2, i}$ given, the speed tracking error $e_{2, i}$ and its derivative, acceleration tracking error, $e_{3, i}$ of the leading vehicle are defined as

$$
\left.\begin{array}{l}
e_{2, i}=x_{2, i}-x_{\mathrm{r} 2, i} \\
e_{3, i}=\dot{x}_{2, i}-\dot{x}_{\mathrm{r} 2, i}
\end{array}\right\} .
$$

Thus, a non-singular terminal sliding manifold for the leading vehicle is defined as

$$
s_{i}=e_{3, i}^{w_{i}}-k_{0, i} e_{2, i},
$$


where the sliding manifold parameters satisfy

$$
0<w_{i}=p_{i} / q_{i}<1,
$$

$p_{i}$ and $q_{i}$ are constants of positive odd integers, and $k_{0, i}<0$ is a constant. Thus, the time derivative of the sliding manifold in Eq. (9) is

$$
\dot{s}_{i}=w_{i} e_{3, i}^{w_{i}-1} \dot{e}_{3, i}-k_{0, i} \dot{e}_{2, i}
$$

where $\dot{e}_{3, i}=\ddot{x}_{2, i}-\ddot{x}_{\mathrm{r} 2, i}$ is the vehicle jerk error and jerk of the target speed profiles, and $\ddot{x}_{\mathrm{r} 2, i}$ is the derivative of the target acceleration profile. Define the time derivative of the total resistance as

$$
\begin{aligned}
& \dot{f}_{\mathrm{R}, i}=\boldsymbol{\theta}_{1, i} \boldsymbol{\zeta}_{1, i}^{\mathrm{T}}(x), \\
& \boldsymbol{\theta}_{1, i}=\left(\dot{f}_{\mathrm{g}, i}, m_{i} c_{1, i}, 2 m_{i} c_{2, i}\right), \\
& \boldsymbol{\zeta}_{1, i}(x)=\left(1, \dot{x}_{2, i}, x_{2, i} \dot{x}_{2, i}\right) .
\end{aligned}
$$

Then from Eq. (6), the jerk of the leading vehicle is

$$
\ddot{x}_{2, i}=\frac{\dot{u}_{\mathrm{e}, i}-\boldsymbol{\theta}_{1, i} \boldsymbol{\zeta}_{1, i}^{\mathrm{T}}(x)}{m_{i}},
$$

and the actual driving force that the engine outputs is

$$
u_{\mathrm{e}, i}=m_{i} \dot{x}_{2, i}+\boldsymbol{\theta}_{i} \boldsymbol{\zeta}_{i}^{\mathrm{T}}(x) .
$$

By substituting Eqs. (7) and (12) into Eq. (11), we obtain

$$
\begin{aligned}
\ddot{x}_{2, i}= & \frac{1}{m_{i}}\left[\frac{1}{T_{i}} u_{\mathrm{c}, i}-\frac{1}{T_{i}}\left(m_{i} \dot{x}_{2, i}+\boldsymbol{\theta}_{i} \boldsymbol{\zeta}_{i}^{\mathrm{T}}(x)\right)-\right. \\
& \left.\boldsymbol{\theta}_{1, i} \boldsymbol{\zeta}_{1, i}^{\mathrm{T}}(x)\right] .
\end{aligned}
$$

Then, Eq. (10) can be rewritten as

$$
\begin{gathered}
\dot{s}_{i}=w_{i} e_{3, i}^{w_{i}-1}\left\{\frac { 1 } { m _ { i } } \left[\frac{1}{T_{i}} u_{\mathrm{c}, i}-\frac{1}{T_{i}}\left(m_{i} \dot{x}_{2, i}+\boldsymbol{\theta}_{i} \boldsymbol{\zeta}_{i}^{\mathrm{T}}(x)\right)-\right.\right. \\
\left.\left.\boldsymbol{\theta}_{1, i} \boldsymbol{\zeta}_{1, i}^{\mathrm{T}}(x)\right]-\ddot{x}_{\mathrm{r} 2, i}\right\}-k_{0, i} e_{3, i} .
\end{gathered}
$$

Based on the nature of the SMC, the control law includes two terms:

$$
u_{\mathrm{c}, i}=u_{\mathrm{eq}, i}+u_{\mathrm{sw}, i}
$$

where $u_{\mathrm{eq}, i}$ is the equivalent control law for the leading vehicle and $u_{\mathrm{sw}, i}$ is the nonlinear switching term. By deriving from $\dot{s}_{i}=0$, the equivalent control law is designed as

$$
\begin{gathered}
u_{\mathrm{eq}, i}=m_{i} \dot{x}_{2, i}+\boldsymbol{\theta}_{i} \boldsymbol{\zeta}_{i}^{\mathrm{T}}(x)+T_{i} \boldsymbol{\theta}_{1, i} \boldsymbol{\zeta}_{1, i}^{\mathrm{T}}(x)+ \\
T_{i} m_{i} \ddot{x}_{\mathrm{r} 2, i}+\frac{T_{i} m_{i} k_{0, i} e_{3, i}^{2-w_{i}}}{w_{i}}
\end{gathered}
$$

and a constant rate reaching law is adopted here for the nonlinear switching term:

$$
u_{\mathrm{sw}, i}=-K_{i} \operatorname{sgn}\left(s_{i}\right),
$$

where $K_{i}>0$ is a parameter greater than the norm bound of the total matched disturbance, satisfying the condition $K_{i} \geqslant\left|\bar{d}_{i}\right|$.

\section{Proof:}

We choose the Lyapunov candidate function for the leading vehicle as

$$
V_{i}=\frac{1}{2} T_{i} m_{i} s_{i}^{2} .
$$

Then, the time derivative of Eq. (18) is

$$
\dot{V}_{i}=T_{i} m_{i} s_{i} \dot{s}_{i}
$$

Then, by substituting Eq. (14) into Eq. (19), the time derivative of the Lyapunov candidate function can be changed to

$$
\begin{aligned}
\dot{V}_{i}= & s_{i}\left\{w _ { i } e _ { 3 , i } ^ { w _ { i } - 1 } \left[u_{\mathrm{c}, i}-m_{i} \dot{x}_{2, i}-\boldsymbol{\theta} \boldsymbol{\zeta}^{\mathrm{T}}(x)-\right.\right. \\
& \left.\left.T_{i} \boldsymbol{\theta}_{1} \boldsymbol{\zeta}_{1}^{\mathrm{T}}(x)-T_{i} m_{i} \ddot{x}_{\mathrm{r} 2, i}\right]-T_{i} m_{i} k_{0, i} e_{3, i}\right\} .
\end{aligned}
$$

Substituting the designed control law Eq. (15) into Eq. (20), we obtain

$$
\dot{V}_{i}=-K_{i} w_{i} e_{3, i}^{w_{i}-1} \operatorname{sgn}\left(s_{i}\right) s_{i}
$$

where $e_{3, i}^{w_{i}-1}>0$ for $\forall e_{3, i} \neq 0$ owing to the odd integers $p_{i}$ and $q_{i}$, and $\operatorname{sgn}\left(s_{i}\right) s_{i}=\left|s_{i}\right|>0$ for $\forall s_{i} \neq 0$. Thus, $\dot{V}_{i}<0$, the system is stable and can reach the sliding manifold $s_{i}=0$ in a finite time.

\section{End of Proof.}

2.2.2 Parameter Adaptation for Leading Vehicle

Owing to model uncertainties, the parameter adaptive law is designed as follows. Based on the control law designed in Eq. (15), two auxiliary variables, $\boldsymbol{\mu}_{\theta, i}=T_{i} \boldsymbol{\theta}_{1, i}$ and $\mu_{m, i}=T_{i} m_{i}$ are defined. The parameters for the adaptive updates are chosen as follows:

$$
\left.\begin{array}{l}
m_{i}=\hat{m}_{i}+\Delta m_{i} \\
\boldsymbol{\theta}_{i}=\hat{\boldsymbol{\theta}}_{i}+\Delta \boldsymbol{\theta}_{i} \\
\boldsymbol{\mu}_{\theta, i}=\hat{\boldsymbol{\mu}}_{\theta, i}+\Delta \boldsymbol{\mu}_{\theta, i} \\
\mu_{m, i}=\hat{\mu}_{m, i}+\Delta \mu_{m, i}
\end{array}\right\}
$$

where $\hat{m}_{i}, \hat{\boldsymbol{\theta}}_{i}, \hat{\boldsymbol{\mu}}_{\theta, i}$, and $\hat{\mu}_{m, i}$ are the estimated values, and $\Delta m_{i}, \Delta \boldsymbol{\theta}_{i}, \Delta \boldsymbol{\mu}_{\theta, i}$, and $\Delta \mu_{m, i}$ are the errors between the estimations and their real values. Then, the designed control law can be rewritten based on the parameter estimation as

$$
\begin{aligned}
u_{\mathrm{c}, i}= & \hat{m}_{i} \dot{x}_{2, i}+\hat{\boldsymbol{\theta}}_{i} \boldsymbol{\zeta}_{i}^{\mathrm{T}}(x)+\hat{\boldsymbol{\mu}}_{\theta, i} \boldsymbol{\zeta}_{1, i}^{\mathrm{T}}(x)+ \\
& \hat{\mu}_{m, i} \ddot{x}_{\mathrm{r} 2, i}+\frac{\hat{\mu}_{m, i} k_{0, i} e_{3, i}^{2-w_{i}}}{w_{i}}-K_{i} \operatorname{sgn}\left(s_{i}\right) .
\end{aligned}
$$

Choose the new Lyapunov candidate function with parameter uncertainties:

$$
\begin{aligned}
V_{i}= & \frac{1}{2} T_{i} m_{i} s_{i}^{2}+\frac{1}{2} \lambda_{m, i}^{-1}(\Delta m)^{2}+\frac{1}{2} \Delta \boldsymbol{\theta}_{i} \boldsymbol{\lambda}_{\theta, i}^{-1}\left(\Delta \boldsymbol{\theta}_{i}\right)^{\mathrm{T}}+ \\
& \frac{1}{2} \Delta \boldsymbol{\mu}_{\theta, i} \boldsymbol{\lambda}_{\mu \theta, i}^{-1}\left(\Delta \boldsymbol{\mu}_{\theta, i}\right)^{\mathrm{T}}+\frac{1}{2} \lambda_{\mu m, i}^{-1}\left(\Delta \mu_{m, i}\right)^{2}
\end{aligned}
$$


where $\lambda_{m, i}, \quad \boldsymbol{\lambda}_{\theta, i}=\operatorname{diag}\left(\lambda_{\theta 1, i}, \lambda_{\theta 2, i}, \lambda_{\theta 3, i}\right), \quad \boldsymbol{\lambda}_{\mu \theta, i}=$ $\operatorname{diag}\left(\lambda_{\mu \theta 1, i}, \lambda_{\mu \theta 2, i}, \lambda_{\mu \theta 3, i}\right)$, and $\lambda_{\mu m, i}$ are the designed constants of the adaptive learning rate. Then, the time derivative of Eq. (24) is

$$
\begin{aligned}
\dot{V}_{i}= & T_{i} m_{i} s_{i} \dot{s}_{i}+\lambda_{m, i}^{-1} \Delta m_{i} \Delta \dot{m}_{i}+\Delta \dot{\boldsymbol{\theta}}_{i} \boldsymbol{\lambda}_{\theta, i}^{-1}\left(\Delta \boldsymbol{\theta}_{i}\right)^{\mathrm{T}}+ \\
& \Delta \dot{\boldsymbol{\mu}}_{\theta, i} \boldsymbol{\lambda}_{\mu \theta, i}^{-1}\left(\Delta \boldsymbol{\mu}_{\theta, i}\right)^{\mathrm{T}}+\lambda_{\mu m, i}^{-1} \Delta \mu_{m, i} \Delta \dot{\mu}_{m, i} .
\end{aligned}
$$

Considering the time derivative of the sliding manifold in Eq. (14), we obtain

$$
\begin{aligned}
T_{i} m_{i} \dot{s}_{i}= & w_{i} e_{3, i}^{w_{i}-1}\left[u_{\mathrm{c}, i}-\left(\hat{m}_{i}+\Delta m_{i}\right) \dot{x}_{2, i}-\right. \\
& \left(\hat{\boldsymbol{\theta}}_{i}+\Delta \boldsymbol{\theta}_{i}\right) \boldsymbol{\zeta}_{i}^{\mathrm{T}}(x)-\left(\hat{\boldsymbol{\mu}}_{\theta, i}+\Delta \boldsymbol{\mu}_{\theta, i}\right) \boldsymbol{\zeta}_{1, i}^{\mathrm{T}}(x)- \\
& \left.\left(\hat{\mu}_{m, i}+\Delta \mu_{m, i}\right) \ddot{x}_{\mathrm{r} 2, i}\right]- \\
& \left(\hat{\mu}_{m, i}+\Delta \mu_{m, i}\right) k_{0, i} e_{3, i}
\end{aligned}
$$

Then, substituting Eqs. (23) and (26) into Eq. (25), we obtain

$$
\begin{aligned}
\dot{V}_{i}= & s_{i}\left\{w _ { i } e _ { 3 , i } ^ { w _ { i } - 1 } \left[-\Delta m_{i} \dot{x}_{2, i}-\Delta \boldsymbol{\theta}_{i} \boldsymbol{\zeta}_{i}^{\mathrm{T}}(x)-\right.\right. \\
& \left.\Delta \boldsymbol{\mu}_{\theta, i} \boldsymbol{\zeta}_{1, i}^{\mathrm{T}}(x)-\Delta \mu_{m, i} \ddot{x}_{\mathrm{r} 2, i}-K_{i} \operatorname{sgn}\left(s_{i}\right)\right]- \\
& \left.\Delta \mu_{m, i} k_{0, i} e_{3, i}\right\}+\lambda_{m, i}^{-1} \Delta m_{i} \Delta \dot{m}_{i}+ \\
& \Delta \dot{\boldsymbol{\theta}}_{i} \boldsymbol{\lambda}_{\theta, i}^{-1}\left(\Delta \boldsymbol{\theta}_{i}\right)^{\mathrm{T}}+\Delta \dot{\boldsymbol{\mu}}_{\theta, i} \boldsymbol{\lambda}_{\mu \theta, i}^{-1}\left(\Delta \boldsymbol{\mu}_{\theta, i}\right)^{\mathrm{T}}+ \\
& \lambda_{m, i}^{-1} \Delta \mu_{m, i} \Delta \dot{\mu}_{m, i} .
\end{aligned}
$$

Note that $\Delta \dot{m}_{i}=-\dot{\hat{m}}_{i}, \Delta \dot{\boldsymbol{\theta}}_{i}=-\dot{\hat{\boldsymbol{\theta}}}_{i}, \Delta \dot{\boldsymbol{\mu}}_{\theta, i}=$ $-\dot{\hat{\boldsymbol{\mu}}}_{\theta, i}$, and $\Delta \dot{\mu}_{m, i}=-\dot{\hat{\mu}}_{m, i}$, and substituting them into Eq. (27), we obtain

$$
\begin{aligned}
\dot{V}_{i}= & -s_{i} w_{i} e_{3, i}^{w_{i}-1} K_{i} \operatorname{sgn}\left(s_{i}\right)- \\
& \Delta m_{i}\left(s_{i} w_{i} e_{3, i}^{w_{i}-1} \dot{x}_{2, i}+\lambda_{m, i}^{-1} \dot{\hat{m}}_{i}\right)- \\
& \Delta \boldsymbol{\theta}_{i}\left(s_{i} w_{i} e_{3, i}^{w_{i}-1} \boldsymbol{\zeta}_{i}^{\mathrm{T}}(x)+\boldsymbol{\lambda}_{\theta, i}^{-1} \dot{\hat{\boldsymbol{\theta}}}_{i}\right)- \\
& \Delta \boldsymbol{\mu}_{\theta, i}\left(s_{i} w_{i} e_{3, i}^{w_{i}-1} \boldsymbol{\zeta}_{1, i}^{\mathrm{T}}(x)+\boldsymbol{\lambda}_{\mu \theta, i}^{-1} \dot{\hat{\boldsymbol{\mu}}}_{\theta, i}\right)- \\
& \Delta \mu_{m, i}\left(s_{i} w_{i} e_{3, i}^{w_{i}-1} \ddot{x}_{\mathrm{r} 2, i}+\right. \\
& \left.s_{i} k_{0, i} e_{3, i}+\lambda_{\mu m, i}^{-1} \dot{\hat{\mu}}_{m, i}\right) .
\end{aligned}
$$

Thus, the adaptive law is designed as

$$
\left.\begin{array}{l}
\dot{\hat{m}}_{i}=-\lambda_{m, i} s_{i} w_{i} e_{3, i}^{w_{i}-1} \dot{x}_{2, i} \\
\dot{\hat{\boldsymbol{\theta}}}_{i}=-\boldsymbol{\lambda}_{\theta, i} s_{i} w_{i} e_{3, i}^{w_{i}-1} \boldsymbol{\zeta}_{i}^{\mathrm{T}}(x) \\
\dot{\hat{\boldsymbol{\mu}}}_{\theta, i}=-\boldsymbol{\lambda}_{\mu \theta, i} s_{i} w_{i} e_{3, i}^{w_{i}-1} \boldsymbol{\zeta}_{1, i}^{\mathrm{T}}(x) \\
\dot{\hat{\mu}}_{m, i}=-\lambda_{\mu m, i}\left(s_{i} w_{i} e_{3, i}^{w_{i}-1} \ddot{x}_{\mathrm{r} 2, i}+s_{i} k_{0, i} e_{3, i}\right)
\end{array}\right\}
$$

and the time derivative of the Lyapunov candidate function becomes

$$
\begin{aligned}
\dot{V}_{i}= & -s_{i} w_{i} e_{3, i}^{w_{i}-1} K_{i} \operatorname{sgn}\left(s_{i}\right)= \\
& -w_{i} e_{3, i}^{w_{i}-1} K_{i}\left|s_{i}\right|<0,
\end{aligned}
$$

which guarantees the stability of the system.

\subsection{Cooperative Controller for Following Vehi- cles}

Because the sliding manifold designed for the leading vehicle in Eq. (9) with the inner stability derived from Eqs. (18) - (21) cannot guarantee strong string stability, we introduce another NTSMC and its associated parameter adaption laws for the following vehicles in the platoon to ensure strong string stability with a nonlinear sliding manifold based on the modified input error idea.

\subsubsection{Control Law Design for Following Vehicles}

For the following vehicles $(i=2,3, \cdots, n)$, based on the same three-order model, Eqs. (6) and (7), and with a D-B reference position profile and real-time position information from the known leading, preceding, and last vehicles, the modified position error from the ego ( $i$ th) vehicle to its preceding vehicle is defined as

$$
\sigma_{1, i}=x_{1, i}-x_{1, i-1}-\left(x_{\mathrm{r} 1, i}-x_{\mathrm{r} 1, i-1}\right) .
$$

Similarly, we have the modified position error between the ego vehicle and leading vehicle as

$$
\sigma_{1,(1, i)}=x_{1, i}-x_{1,1}-\left(x_{\mathrm{r} 1, i}-x_{\mathrm{r} 1,1}\right),
$$

and the error between the ego vehicle and last vehicle of the platoon as

$$
\sigma_{1,(n, i)}=x_{1, n}-x_{1, i}-\left(x_{\mathrm{r} 1, n}-x_{\mathrm{r} 1, i}\right) .
$$

Then, we define a linear combination of Eqs. (31) (33) as the input error $z_{1, i}$ for the closed-loop system for the following vehicles:

$$
z_{1, i}=\sigma_{1, i}+\alpha \sigma_{1,(1, i)}+\beta \sigma_{1,(n, i)}
$$

where $\alpha>\beta \geqslant 0$ are the coefficients of the linear coupling. It is worth noting that the case of $\beta=0$ indicates that the transformed input error regards only the ego vehicle itself and the leading vehicle, which also means that the optional communication link with the last vehicle mentioned in Subsection 1.1 is not activated.

Based on the same idea as in controller design for the leading vehicle, a non-singular terminal sliding manifold is also adopted and designed with new input errors as

$$
s_{i}=z_{2, i}^{w_{i}}-k_{0} z_{1, i}
$$

where $z_{2, i}=\dot{z}_{1, i}$ is the time derivative of the input error $z_{1, i}$. Then, we define an auxiliary variable $\pi_{i}$ and its derivative $\dot{\pi}_{i}$ as

$$
\left.\begin{array}{rl}
\pi_{i}= & \alpha x_{2,1}+x_{2, i-1}-\beta x_{2, n}-\alpha x_{\mathrm{r} 2,1}- \\
& x_{\mathrm{r} 2, i-1}+(1+\alpha-\beta) x_{\mathrm{r} 2, i}+\beta x_{\mathrm{r} 2, n} \\
\dot{\pi}_{i}= & \alpha \dot{x}_{2,1}+\dot{x}_{2, i-1}-\beta \dot{x}_{2, n}-\alpha \dot{x}_{\mathrm{r} 2,1}- \\
& \dot{x}_{\mathrm{r} 2, i-1}+(1+\alpha-\beta) \dot{x}_{\mathrm{r} 2, i}+\beta \dot{x}_{\mathrm{r} 2, n}
\end{array}\right\} .
$$


Thus, we have

$$
\left.\begin{array}{l}
z_{2, i}=(1+\alpha-\beta) x_{2, i}-\pi_{i} \\
\dot{z}_{2, i}=(1+\alpha-\beta) \dot{x}_{2, i}-\dot{\pi}_{i}
\end{array}\right\} .
$$

Then, the time derivative of the sliding manifold for following vehicles is

$$
\dot{s}_{i}=w_{i} z_{2, i}^{w_{i}-1}\left[(1+\alpha-\beta) \dot{x}_{2, i}-\dot{\pi}_{i}\right]-k_{0, i} z_{2, i} .
$$

Taking steps that are similar to those described in Subsection 2.2, we can obtain the time derivative of the sliding manifold:

$$
\begin{gathered}
\dot{s}_{i}=w_{i} z_{2, i}^{w_{i}-1}\left\{( 1 + \alpha - \beta ) \frac { 1 } { m _ { i } } \left[u_{\mathrm{c}, i}-T_{i}\left(m_{i} \ddot{x}_{2, i}+\right.\right.\right. \\
\left.\left.\left.\boldsymbol{\theta}_{1, i} \boldsymbol{\zeta}_{1, i}^{\mathrm{T}}(x)\right)-\boldsymbol{\theta}_{i} \boldsymbol{\zeta}_{i}^{\mathrm{T}}(x)\right]-\dot{\pi}_{i}\right\}-k_{0, i} z_{2, i} .
\end{gathered}
$$

Therefore, the equivalent control law for the sliding manifold of the following vehicles, designed by assuming that $\dot{s}_{i}=0$ is

$$
\begin{aligned}
u_{\mathrm{eq}, i}= & T_{i} m_{i} \ddot{x}_{2, i}+T_{i} \boldsymbol{\theta}_{1, i} \boldsymbol{\zeta}_{1, i}^{\mathrm{T}}(x)+\boldsymbol{\theta}_{i} \boldsymbol{\zeta}_{i}^{\mathrm{T}}(x)+ \\
& \frac{m_{i} \dot{\pi}_{i}}{1+\alpha-\beta}+\frac{m_{i} k_{0, i} z_{2, i}^{2-w_{i}}}{(1+\alpha-\beta) w_{i}}
\end{aligned}
$$

The constant rate reaching law is also chosen as the nonlinear switch part of the control law, which is

$$
u_{\mathrm{sw}, i}=-K_{i} \operatorname{sgn}\left(s_{i}\right),
$$

where $K_{i}>0$ is a parameter greater than the norm bound of the total matched disturbance, satisfying the condition $K_{i} \geqslant\left|\bar{d}_{i}\right|$.

Thus, the control law is designed as the summation of the equivalent control law in Eq. (40) and nonlinear switch part Eq. (41) as

$$
u_{\mathrm{c}, i}=u_{\mathrm{eq}, i}+u_{\mathrm{sw}, i} .
$$

\section{Proof:}

Like the proof in Subsection 2.2.1, a Lyapunov candidate function is chosen as

$$
V_{i}=\frac{1}{2} m_{i} s_{i}^{2},
$$

and the time derivative of Eq. (43) is

$$
\dot{V}_{i}=m_{i} s_{i} \dot{s}_{i}
$$

By substituting Eq. (39) into Eq. (44), the time derivative of the Lyapunov candidate function can be changed to

$$
\begin{gathered}
\dot{V}_{i}=s_{i}\left(w _ { i } z _ { 2 , i } ^ { w _ { i } - 1 } \left\{( 1 + \alpha - \beta ) \frac { 1 } { m _ { i } } \left[u_{\mathrm{c}, i}-T_{i}\left(m_{i} \ddot{x}_{2, i}+\right.\right.\right.\right. \\
\left.\left.\left.\left.\boldsymbol{\theta}_{1, i} \boldsymbol{\zeta}_{1, i}^{\mathrm{T}}(x)\right)-\boldsymbol{\theta}_{i} \boldsymbol{\zeta}_{i}^{\mathrm{T}}(x)\right]-\dot{\pi}_{i}\right\}-k_{0, i} z_{2, i}\right),
\end{gathered}
$$

and by substituting the designed control law in Eq. (42) into Eq. (45), the time derivative of Eq. (43) can be obtained as

$$
\dot{V}_{i}=-K_{i} w_{i} z_{2, i}^{w_{i}-1} \operatorname{sgn}\left(s_{i}\right) s_{i}
$$

where $z_{2, i}^{w_{i}-1}>0$ for $\forall z_{2, i} \neq 0$, owing to the odd integers $p_{i}$ and $q_{i}$, and $\operatorname{sgn}\left(s_{i}\right) s_{i}=\left|s_{i}\right|>0$ for $\forall s_{i} \neq 0$. Thus $\dot{V}_{i}<0$, the system is stable and can reach the sliding manifold $s_{i}=0$ in a finite time.

\section{End of Proof.}

2.3.2 Parameter Adaptation for Following Vehicles

Similarly, parameter adaptation is designed for the following vehicles. Using the same definition of parameter uncertainties as in Eq. (22), the new Lyapunov candidate function is designed as

$$
\begin{aligned}
V_{i}= & \frac{1}{2} m_{i} s_{i}^{2}+\frac{1}{2} \lambda_{m, i}^{-1}\left(\Delta m_{i}\right)^{2}+\frac{1}{2} \Delta \boldsymbol{\theta}_{i} \boldsymbol{\lambda}_{\theta, i}^{-1}\left(\Delta \boldsymbol{\theta}_{i}\right)^{\mathrm{T}}+ \\
& \frac{1}{2} \Delta \boldsymbol{\mu}_{\theta, i} \boldsymbol{\lambda}_{\mu \theta, i}^{-1}\left(\Delta \boldsymbol{\mu}_{\theta, i}\right)^{\mathrm{T}}+\frac{1}{2} \lambda_{\mu m, i}^{-1}\left(\Delta \mu_{m, i}\right)^{2},
\end{aligned}
$$

where $\lambda_{m, i}, \boldsymbol{\lambda}_{\theta, i}, \boldsymbol{\lambda}_{\mu \theta, i}$, and $\lambda_{\mu m, i}$ are the parameters of the adaptive learning rate with the same definition as in Subsection 2.2.2 and the proper dimension.

Then, the time derivative of Eq. (47) becomes

$$
\begin{aligned}
\dot{V}_{i}= & m_{i} s_{i} \dot{s}_{i}+\lambda_{m, i}^{-1} \Delta m_{i} \Delta \dot{m}_{i}+\Delta \dot{\boldsymbol{\theta}}_{i} \boldsymbol{\lambda}_{\theta, i}^{-1}\left(\Delta \boldsymbol{\theta}_{i}\right)^{\mathrm{T}}+ \\
& \Delta \dot{\boldsymbol{\mu}}_{\theta, i} \boldsymbol{\lambda}_{\mu \theta, i}^{-1}\left(\Delta \boldsymbol{\mu}_{\theta, i}\right)^{\mathrm{T}}+\lambda_{\mu m, i}^{-1} \Delta \mu_{m, i} \Delta \dot{\mu}_{m, i} .
\end{aligned}
$$

We consider the time derivative of the sliding manifold with parameter uncertainties:

$$
\begin{aligned}
m_{i} \dot{s}_{i}= & w_{i} z_{2, i}^{w_{i}-1}\left\{( 1 + \alpha - \beta ) \left[u_{c, i}-\left(\hat{\mu}_{m, i}+\Delta \mu_{m, i}\right) \ddot{x}_{2, i}-\right.\right. \\
& \left.\left(\hat{\boldsymbol{\mu}}_{\theta, i}+\Delta \boldsymbol{\mu}_{\theta, i}\right) \boldsymbol{\zeta}_{1, i}^{\mathrm{T}}(x)-\left(\hat{\boldsymbol{\theta}}_{i}+\Delta \boldsymbol{\theta}_{i}\right) \boldsymbol{\zeta}_{i}^{\mathrm{T}}(x)\right]- \\
& \left.\left(\hat{m}_{i}+\Delta m_{i}\right) \dot{\pi}_{i}\right\}-\left(\hat{m}_{i}+\Delta m_{i}\right) k_{0, i} z_{2, i}
\end{aligned}
$$

Then, the designed control law with parameter uncertainties is

$$
\begin{aligned}
u_{\mathrm{c}, i}= & \hat{\mu}_{m, i} \ddot{x}_{2, i}+\hat{\boldsymbol{\mu}}_{\theta, i} \boldsymbol{\zeta}_{1, i}^{\mathrm{T}}(x)+\hat{\boldsymbol{\theta}}_{i} \boldsymbol{\zeta}_{i}^{\mathrm{T}}(x)+ \\
& \frac{\hat{m}_{i} \dot{\pi}_{i}}{1+\alpha-\beta}+\frac{\hat{m}_{i} k_{0, i} z_{2, i}^{2-w_{i}}}{(1+\alpha-\beta) w_{i}}-K_{i} \operatorname{sgn}\left(s_{i}\right) .
\end{aligned}
$$

We substitute Eqs. (49) and (50) into Eq. (48). Then, the time derivative of Eq. (47) can be obtained:

$$
\begin{aligned}
\dot{V}_{i}= & s_{i}\left[w _ { i } z _ { 2 , i } ^ { w _ { i } - 1 } \left(-\frac{\Delta \mu_{m, i} \dot{x}_{2, i}}{1+\alpha-\beta}-\frac{\Delta \boldsymbol{\theta}_{i} \boldsymbol{\zeta}_{i}^{\mathrm{T}}(x)}{1+\alpha-\beta}-\right.\right. \\
& \left.\frac{\Delta \boldsymbol{\mu}_{\theta, i} \boldsymbol{\zeta}_{1, i}^{\mathrm{T}}(x)}{1+\alpha-\beta}-\Delta m_{i} \dot{\pi}_{i}-K_{i} \operatorname{sgn}\left(s_{i}\right)\right)- \\
& \left.\Delta m_{i} k_{0, i} z_{2, i}\right]+\lambda_{m, i}^{-1} \Delta m_{i} \Delta \dot{m}_{i}+\Delta \dot{\boldsymbol{\theta}}_{i} \boldsymbol{\lambda}_{\theta, i}^{-1}\left(\Delta \boldsymbol{\theta}_{i}\right)^{\mathrm{T}}+ \\
& \Delta \dot{\boldsymbol{\mu}}_{\theta, i} \boldsymbol{\lambda}_{\mu \theta, i}^{-1}\left(\Delta \boldsymbol{\mu}_{\theta, i}\right)^{\mathrm{T}}+\lambda_{\mu m, i}^{-1} \Delta \mu_{m, i} \Delta \dot{\mu}_{m, i} .
\end{aligned}
$$


Similarly, note that $\Delta \dot{m}_{i}=-\dot{\hat{m}}_{i}, \Delta \dot{\boldsymbol{\theta}}_{i}=-\dot{\hat{\boldsymbol{\theta}}}_{i}$, $\Delta \dot{\boldsymbol{\mu}}_{\theta, i}=-\dot{\hat{\boldsymbol{\mu}}}_{\theta, i}$, and $\Delta \dot{\mu}_{m, i}=-\dot{\hat{\mu}}_{m, i}$, and substituting them into Eq. (51), we obtain

$$
\begin{aligned}
\dot{V}_{i}= & -s_{i} w_{i} z_{2, i}^{w_{i}-1} K_{i} \operatorname{sgn}\left(s_{i}\right)- \\
& \Delta m_{i}\left(s_{i} w_{i} z_{2, i}^{w_{i}-1} \dot{\pi}_{i}+s_{i} k_{0, i} z_{2, i}+\lambda_{m, i}^{-1} \dot{\hat{m}}_{i}\right)- \\
& \Delta \boldsymbol{\theta}_{i}\left(\frac{s_{i} w_{i} z_{2, i}^{w_{i}-1} \boldsymbol{\zeta}_{i}^{\mathrm{T}}(x)}{1+\alpha-\beta}+\boldsymbol{\lambda}_{\theta, i}^{-1} \dot{\hat{\boldsymbol{\theta}}}_{i}\right)- \\
& \Delta \boldsymbol{\mu}_{\theta, i}\left(\frac{s_{i} w_{i} z_{2, i}^{w_{i}-1} \boldsymbol{\zeta}_{1, i}^{\mathrm{T}}(x)}{1+\alpha-\beta}+\boldsymbol{\lambda}_{\mu \theta, i}^{-1} \dot{\hat{\boldsymbol{\mu}}}_{\theta, i}\right)- \\
& \Delta \mu_{m, i}\left(\frac{s_{i} w_{i} z_{2, i}^{w_{i}-1} \dot{x}_{2, i}}{1+\alpha-\beta}+\lambda_{\mu m, i}^{-1} \dot{\hat{\mu}}_{m, i}\right) .
\end{aligned}
$$

Thus, the adaptive law is designed as

$$
\left.\begin{array}{l}
\dot{\hat{m}}_{i}=-\lambda_{m, i}\left(s_{i} w_{i} z_{2, i}^{w_{i}-1} \dot{\pi}_{i}+s_{i} k_{0, i} z_{2, i}\right) \\
\dot{\hat{\boldsymbol{\theta}}}=-\frac{\boldsymbol{\lambda}_{\theta, i} s_{i} w_{i} z_{2, i}^{w_{i}-1} \boldsymbol{\zeta}_{i}^{\mathrm{T}}(x)}{1+\alpha-\beta} \\
\dot{\hat{\boldsymbol{\mu}}}_{\theta}=-\frac{\boldsymbol{\lambda}_{\mu \theta, i} s_{i} w_{i} z_{2, i}^{w_{i}-1} \boldsymbol{\zeta}_{1, i}^{\mathrm{T}}(x)}{1+\alpha-\beta} \\
\dot{\hat{\mu}}_{m}=-\frac{\lambda_{\mu m, i} s_{i} w_{i} z_{2, i}^{w_{i}-1} \dot{x}_{2, i}}{1+\alpha-\beta}
\end{array}\right\} .
$$

Then, the time derivative of the Lyapunov candidate function becomes

$$
\begin{aligned}
\dot{V}_{i}= & -s_{i} w_{i} z_{2, i}^{w_{i}-1} K_{i} \operatorname{sgn}\left(s_{i}\right)= \\
& -w_{i} z_{2, i}^{w_{i}-1} K_{i}\left|s_{i}\right|<0,
\end{aligned}
$$

which guarantees the stability of the system.

\subsection{String Stability}

String stability is a vital requirement for interconnected systems such as vehicular platoons to attenuate state errors in the upstream direction. The definition of strong string stability derived in Ref. [7] is adopted in this study, expressed in such a way that the Laplace transforms of the error propagation $\mathcal{G}_{i}(s)=\frac{E_{i+1}(s)}{E_{i}(s)}$ satisfy $\left\|\mathcal{G}_{i}(s)\right\| \leqslant 1$ for all following vehicles $\forall i \in[2, n]$.

Based on the inner stability in Subsection 2.3, for the following vehicles, the sliding manifold converges to zero in a finite time. Thus, we have

$$
z_{1, i}=\sigma_{1, i}+\alpha \sigma_{1,(1, i)}+\beta \sigma_{1,(n, i)}=0 .
$$

According to the definition of the modified position error, Eqs. (31)-(33), we obtain

$$
\left.\begin{array}{l}
\sigma_{1,(1, i)}-\sigma_{1,(1, i-1)}=\sigma_{1, i} \\
\sigma_{1,(n, i)}-\sigma_{1,(n, i-1)}=-\sigma_{1, i}
\end{array}\right\} .
$$

Inserting Eq. (56) into Eq. (55), we obtain

$$
(1+\alpha-\beta) \sigma_{1, i}+\alpha \sigma_{1,(1, i-1)}+\beta \sigma_{1,(n, i-1)}=0 .
$$

Considering $z_{1, i-1}=\sigma_{1, i-1}+\alpha \sigma_{1,(1, i-1)}+$ $\beta \sigma_{1,(n, i-1)}=0$, we have

$$
\alpha \sigma_{1,(1, i-1)}+\beta \sigma_{1,(n, i-1)}=-\sigma_{1, i-1} .
$$

Then, inserting Eq. (58) into Eq. (57), we obtain

$$
\sigma_{1, i-1}+\alpha \sigma_{1,(1, i-1)}+\beta \sigma_{1,(n, i-1)}=0,
$$

which is

$$
\frac{\sigma_{1, i}}{\sigma_{1, i-1}}=\frac{1}{1+\alpha-\beta} .
$$

If we choose the coupling parameters satisfying $\alpha>$ $\beta \geqslant 0$, then $\frac{1}{1+\alpha-\beta}<1$. Hence, we obtain

$$
\frac{\left\|E\left(\sigma_{1, i}\right)\right\|}{\left\|E\left(\sigma_{1, i-1}\right)\right\|}=\frac{1}{1+\alpha-\beta}<1,
$$

where $E\left(\sigma_{1, i}\right)$ and $E\left(\sigma_{1, i-1}\right)$ are the Laplace transforms of $\sigma_{1, i}$ and, $\sigma_{1, i-1}$, respectively, which guarantees the string stability of the vehicle platoon system.

\section{Numerical Simulation}

In this section, numerical simulations are conducted to illustrate the effectiveness of the developed control schemes. A vehicular platoon with seven CAVs was configured. The parameters of the simulated vehicles were obtained from Ref. [14], with $m_{i}=1607 \mathrm{~kg}, c_{i}=$ $0.414, f_{i}=236.229, T_{i}=0.25 \mathrm{~s}$, and $\tau_{i}=0.3 \mathrm{~s}$. The acceleration and jerk of the vehicles were bounded within $\pm 2 \mathrm{~m} / \mathrm{s}^{2}$ and $\pm 4 \mathrm{~m} / \mathrm{s}^{3}$, respectively. The undulate road profile, target velocity, and target acceleration trajectories for the vehicular platoon are indicated in Fig. 3. We selected the vehicle position and speed with random errors as the initial state for the simulation to test the proposed adaptive NTSMC controller in a more general condition. The initial state of the leading vehicle was set as $\left(x_{1,1}, x_{2,1}\right)=(300,14)$. The states of the other six following vehicles were set as $\left(x_{1,2}, x_{2,2}\right)=(250,13.3)$, $\left(x_{1,3}, x_{2,3}\right)=(200,12.7),\left(x_{1,4}, x_{2,4}\right)=(140,12.0)$, $\left(x_{1,5}, x_{2,5}\right)=(90,11.3),\left(x_{1,6}, x_{2,6}\right)=(40,10.6)$, and $\left(x_{1,7}, x_{2,7}\right)=(0,10.0)$. The time gap between each successive vehicle was selected as $5 \mathrm{~s}$.

The simulation and control cycles were both $0.1 \mathrm{~s}$ and the numerical integrations in the simulation were computed by the Runge-Kutta method. The parameters of the adaptive NTSMC were set to $p_{i}=15, q_{i}=13$, and $k_{0, i}=-0.1$ for the leading vehicle and $k_{0, i}=-0.5$ for the following vehicles. These are based on the requirements for $p_{i}$ and $q_{i}$ to be positive odd integers and the sliding manifold reaching time $t_{\mathrm{r}}$ given from Ref. [25],

$$
t_{\mathrm{r}}=\frac{p_{i}}{-k_{0, i}\left(p_{i}-q_{i}\right)}|e(0)|^{\left(1-\frac{q_{i}}{p_{i}}\right)},
$$




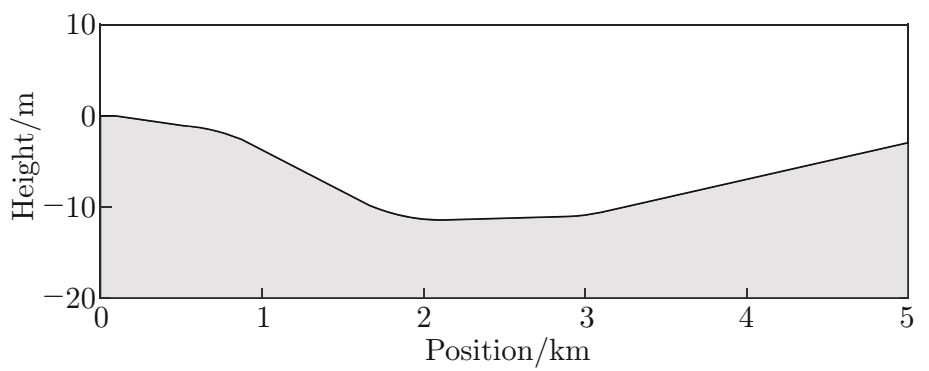

(a) Road vertical profile

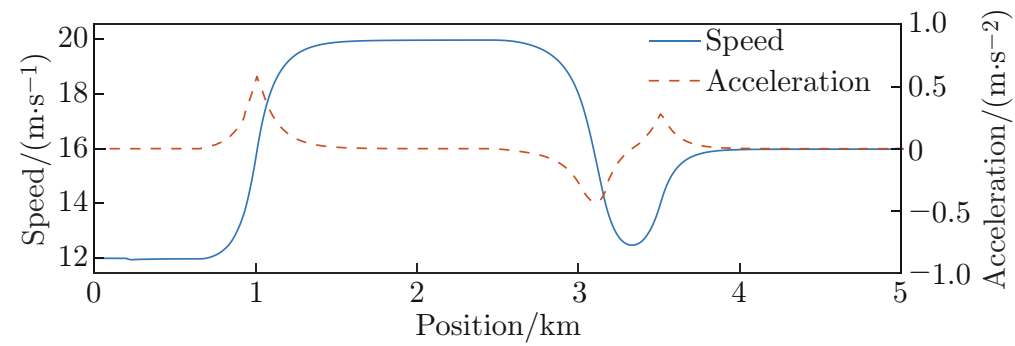

(b) Target speed and acceleration

Fig. 3 Simulation scenario

where $e(0)=e_{2,1}(0)$ is for the leading vehicle, and $e(0)=\sigma_{1, i}(0)$ is for the following vehicles. The switching gain was designed as $K_{i}=m_{i} \bar{d}_{i}=160.7$, based on the bounded acceleration caused by the unknown disturbance, which was assumed to be $\bar{d}_{i}=0.1 \mathrm{~m} / \mathrm{s}^{2}$ multiplied by the vehicle mass. The linear coupling coeffi- cients for the following vehicles were selected as $\alpha=0.9$ and $\beta=0.6$, which satisfy the string stable condition $\alpha>\beta \geqslant 0$. The adaptive learning rates were chosen as $\lambda_{\mathrm{m}}=0.5$ and $\left(\lambda_{\theta 1}, \lambda_{\theta 2}, \lambda_{\theta 3}\right)=(0.005,0.002,0.001)$ based on the magnitude of each parameter.

The simulation results are presented in Figs. $4-7$.

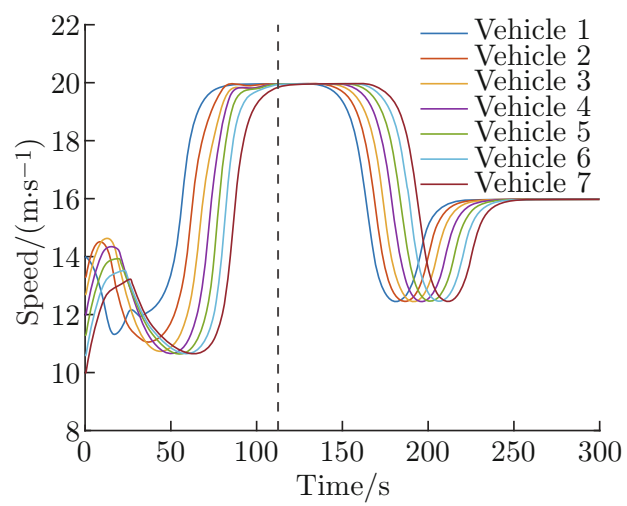

(a) Speed vs. time profiles

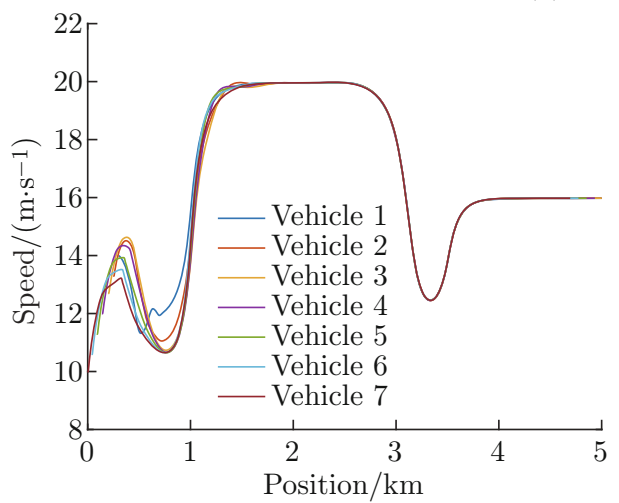

(b) Speed vs. position profiles

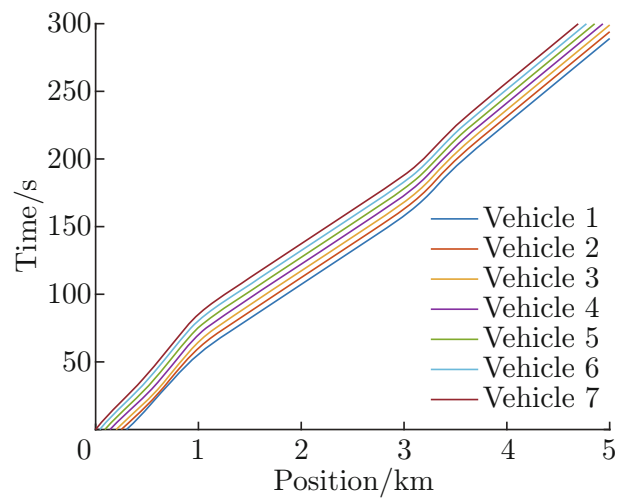

(c) Time vs. position profiles

Fig. 4 Trajectories of vehicular platoon 


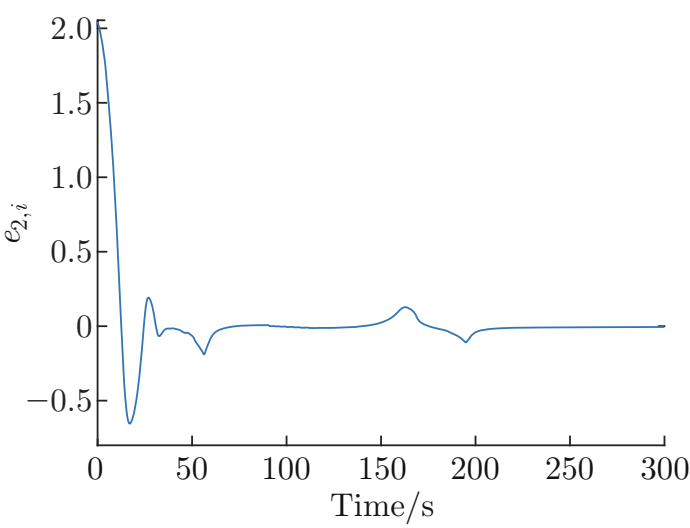

(a) Speed error about target profile

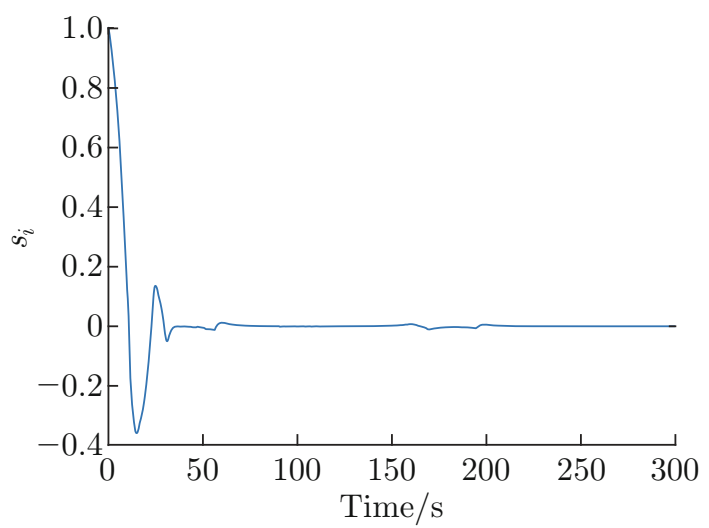

(b) Sliding manifold

Fig. 5 Tracking performance of the leading vehicle

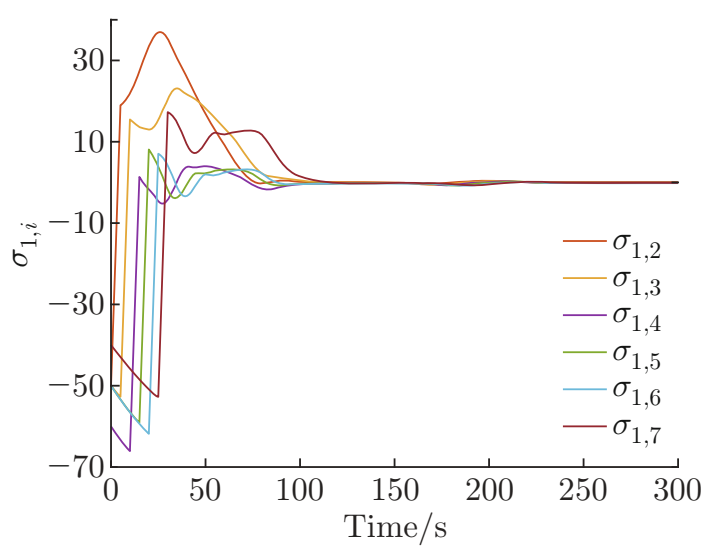

(a) Position errors of following vehicles

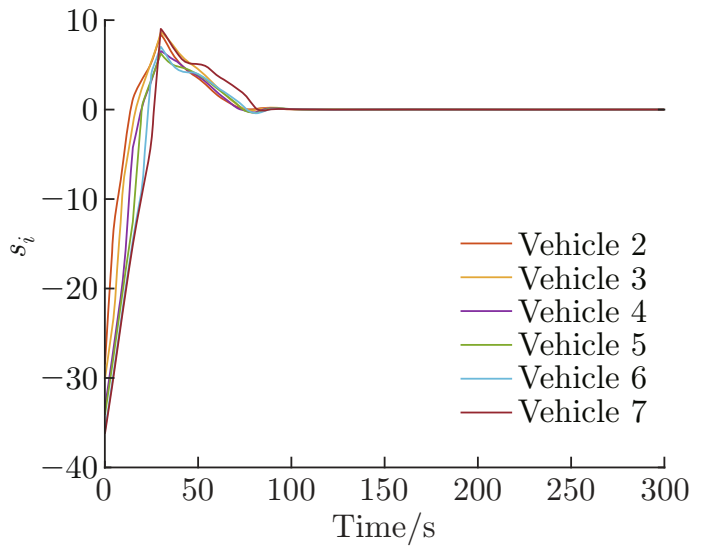

(b) Sliding manifolds

Fig. 6 Tracking performance of following vehicles

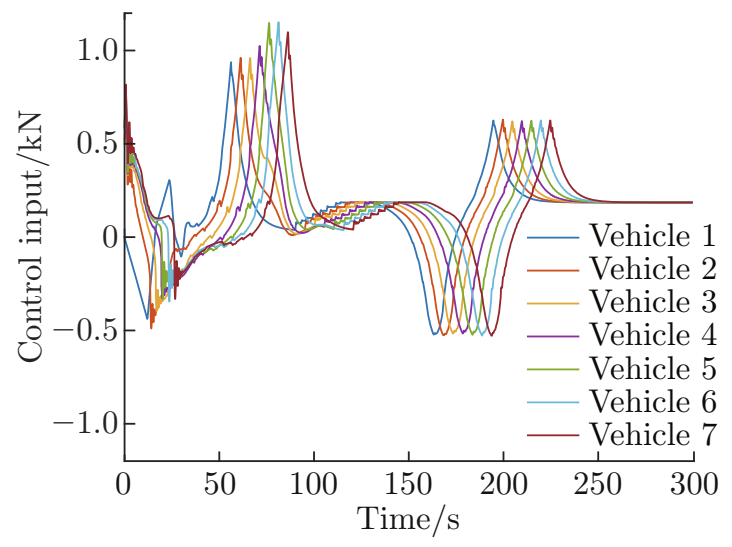

Fig. 7 Control command inputs for all vehicles

The stabilization time (ST), after which the speed error for each vehicle is within a predefined range, is introduced as one of the measures of effectiveness (MOEs). In this study, the threshold of the ST speed error bound was set as $\pm 0.05 \mathrm{~m} / \mathrm{s}$. As indicated in Fig. 4(a), the $\mathrm{ST}$ was $112.5 \mathrm{~s}$, which is represented by the vertical gray dashed line. In Figs.4(b) and 4(c), the velocity and time profiles for each vehicle in the platoon are presented with respect to their position owing to the adopted D-B policy. Stabilized by the proposed adaptive NTSMC controllers, different initial velocity errors converge after $1500 \mathrm{~m}$, as indicated in Fig. 4(b). All vehicle trajectories remained the same in the space domain. Figure 4(c) indicates that the time gaps tended to be consistent with the predefined value, which is reflected by a set of parallel time profiles after the position at $1000 \mathrm{~m}$.

Because different controllers were adopted in the leading and following vehicles, the tracking performances described by the state deviations and sliding manifolds are separately presented in Figs. 5 and 6 in the time domain, presenting the convergence properties of the proposed controllers. The initial speed error of the leading vehicle converged to a zero-state within $30 \mathrm{~s}$, which is faster than the converging process for the following vehicle position errors because the modified position errors of the following vehicles are not only based on ego error, but also considering the error between the leading, preceding, and last vehicles. The sliding manifolds of the following vehicles converged to the zero-state after $100 \mathrm{~s}$. 
The control command inputs for all vehicles are displayed in Fig. 7. It can be observed that the command inputs are smooth and no clear collisions or chattering phenomena occurred. The control input of a vehicle is a time-delay similar to its preceding vehicle after approximately the first $30 \mathrm{~s}$ of the stabilizing process, which consists of the D-B policy adoption for the target profile generation. Thus, it can be perceived that the realized controllers can potentially be used in practice.

\section{Comparison Experiment}

Because studies based on SMC for D-B policy are rare, a scenario with a constant target speed and zero acceleration was adopted for the simulation comparison. In a constant target speed setting, the reference speed and desired inner vehicle distance for the following vehicles exhibit the same behavior under both the CS and D-B policies.

The CSMC method proposed in Ref. [11] was chosen as the comparative following vehicle controller. In this comparison scenario, the constant target speed was set to $16 \mathrm{~m} / \mathrm{s}$ and the total simulation time was $500 \mathrm{~s}$. Other than these two modifications, the initial state

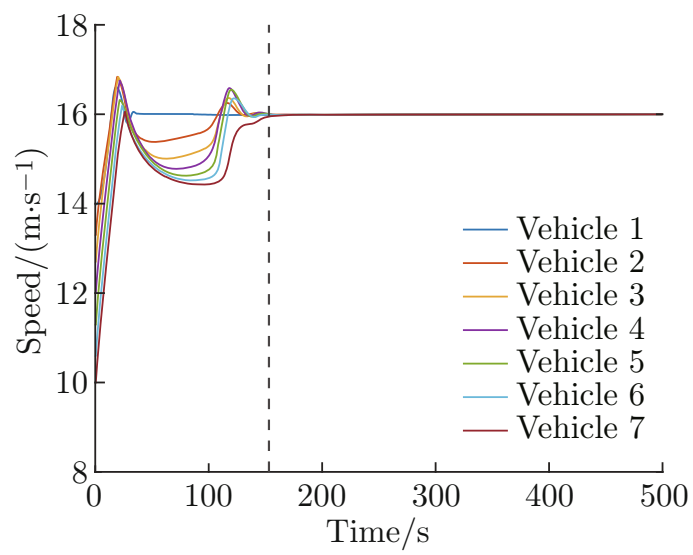

(a) Speed vs. time profiles of each vehicle and other configurations remained unchanged, as described in Section 3. The desired inner vehicle distance was set to $80 \mathrm{~m}$ in the CS policy, which is equivalent to the $5 \mathrm{~s}$ time gap in the $\mathrm{D}$-B policy.

In addition to $\mathrm{ST}$, the maximum speed overshoot (MSO), the integrals of time multiplied by the absolute value of error (ITAEs) for the vehicle speed and acceleration which are defined as

$$
\left.\begin{array}{l}
J_{\text {Speed }}^{\text {(ITAE) }}=\sum_{i=1}^{n} \int_{t_{0}}^{t_{\mathrm{f}}} t\left|e_{2, i}(t)\right| \mathrm{d} t \\
J_{\text {Accel }}^{\text {(ITAE) }}=\sum_{i=1}^{n} \int_{t_{0}}^{t_{\mathrm{f}}} t\left|e_{3, i}(t)\right| \mathrm{d} t
\end{array}\right\},
$$

and the maximum absolute desired distance deviation (MADDD) after ST were chosen as the other MOE indices. In Eq. (62), $t_{0}$ and $t_{\mathrm{f}}$ are the start and end time for the ITEA computation. MADDD is defined as the difference between the inner vehicle distance and desired distance, which was $80 \mathrm{~m}$.

The simulation results of the proposed controller are displayed in Figs. 8 and 9, and the results of the CSMC controller are presented in Figs. 10 and 11.

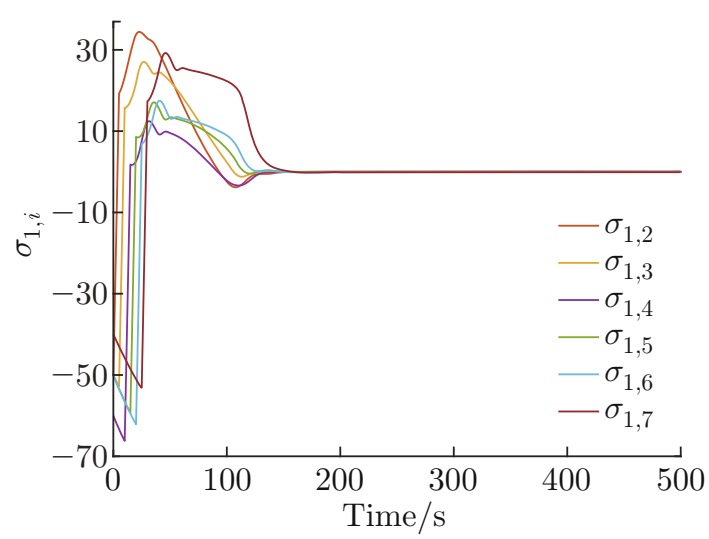

(b) Position errors of following vehicles

Fig. 8 Tracking performance using proposed method

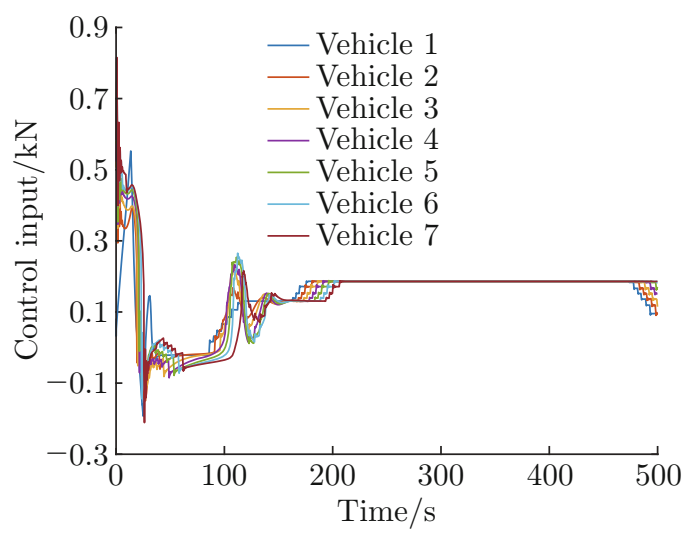

Fig. 9 Control inputs using proposed method
By comparing Figs. 8 and 9 with Figs. 10 and 11, respectively, it can be seen that with the same initial states, the proposed controller required approximately $44.73 \%$ less time to stabilize the vehicle platoon. The speed overshoot was $11.20 \%$ less than that of the CSMC controller, and the maximum steady-state inner vehicle distance deviation was virtually zero for the proposed controller, whereas the CSMC controller had a maximum steady-state error of approximately $1.94 \%$ of the desired distance. The control command inputs using the proposed method were smooth and exhibited no clear chattering phenomena. The five MOE indices listed in Table 1 indicate the performance of the proposed controller. 


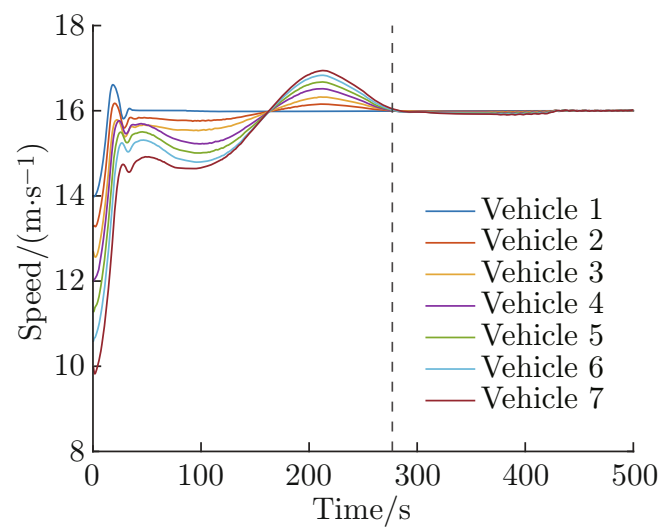

(a) Speed vs. time profiles

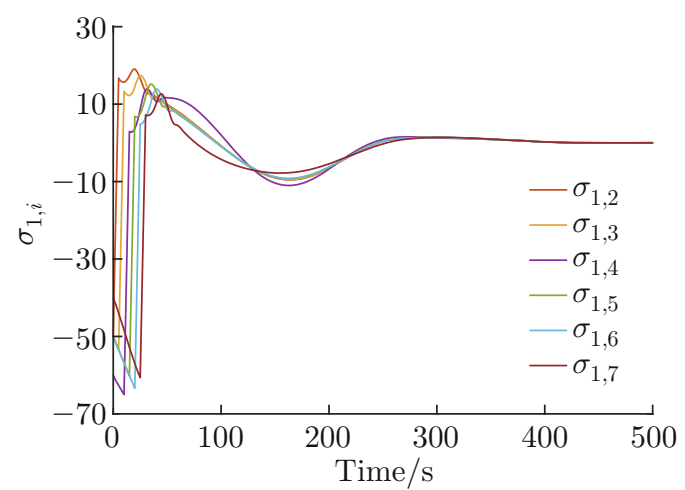

(b) Position errors of following vehicles

Fig. 10 Tracking performance using CSMC

Table 1 Comparison of key performance

\begin{tabular}{cccccc}
\hline Controller & $\mathrm{ST} / \mathrm{s}$ & $J_{\text {Speed }}^{\text {(ITAE) }}$ & $J_{\text {Accel }}^{(\text {ITAE })}$ & $\mathrm{MSO} /\left(\mathrm{m} \cdot \mathrm{s}^{-1}\right)$ & $\mathrm{MADDD} / \mathrm{m}$ \\
\hline Proposed & 153.1 & 42865.142 & 2165.127 & 0.848 & 0.173 \\
CSMC & 277.0 & 109621.406 & 4391.282 & 0.955 & 1.548 \\
\hline
\end{tabular}

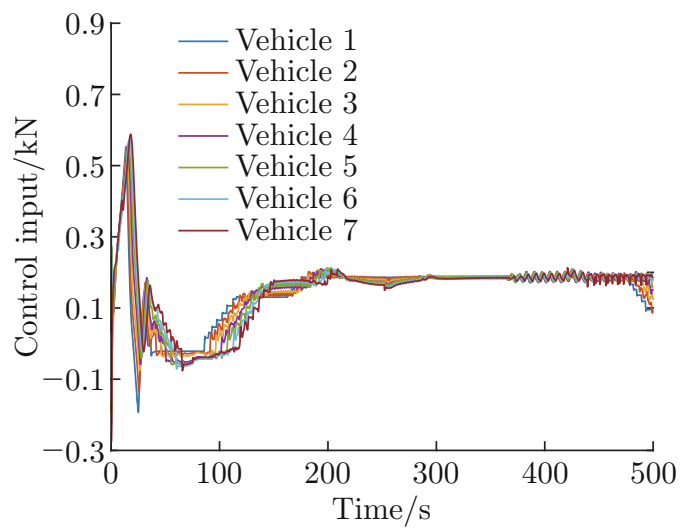

Fig. 11 Control inputs using CSMC

\section{Conclusion}

The CACC control problem in a vehicular platoon was studied. Two new CACC controllers based on the robust adaptive NTSMC approach were proposed for the leading and following vehicles. The D-B policy was adopted to ensure the same target speed tracking in the space domain and target time headway for all vehicles in the platoon. Different control objectives, which were the given speed and acceleration profile tracking for the leading vehicle, and proper distance and headway maintenance for the following vehicles were designed. By considering the engine dynamics modeled as the FOPDT process, a three-order nonlinear vehicle longitudinal dynamics model was adopted. To compensate and eliminate the influence of pure command delay, an
SP consisting of nominal vehicle models with and without time delay was connected in parallel in the control loop. A nonlinear non-singular terminal sliding manifold was designed for the leading vehicle based on its speed and acceleration errors, and the control and parameter adaptive laws were derived by Lyapunov functions that ensured the inner stability and vehicle velocity tracking performance. Using the predecessor-leader following and optional last-to-ego vehicle information topology, the string stability was guaranteed in a similar nonlinear sliding manifold for the following vehicles through a linear coupled position error. Finally, numerical simulation results of a seven-CAV platoon and comparison experiments with a CSMC controller confirmed the effectiveness and outperformance of the proposed approach.

Open Access This article is licensed under a Creative Commons Attribution 4.0 International License, which permits use, sharing, adaptation, distribution and reproduction in any medium or format, as long as you give appropriate credit to the original author(s) and the source, provide a link to the Creative Commons licence, and indicate if changes were made. The images or other third party material in this article are included in the article's Creative Commons licence, unless indicated otherwise in a credit line to the material. If material is not included in the article's Creative Commons licence and your intended use is not permitted by statutory regulation or exceeds the permitted use, you will need to obtain permission directly from the copyright holder. To view a copy of this licence, visit http://creativecommons.org/licenses/by/4.0/. 


\section{References}

[1] ALAM A, BESSELINK B, TURRI V, et al. Heavyduty vehicle platooning for sustainable freight transportation: A cooperative method to enhance safety and efficiency [J]. IEEE Control Systems Magazine, 2015, 35(6): 34-56.

[2] SHLADOVER S E, NOWAKOWSKI C, LU X Y, et al. Cooperative adaptive cruise control: Definitions and operating concepts [J]. Transportation Research Record: Journal of the Transportation Research Board, 2015, 2489(1): 145-152.

[3] WANG Z, BIAN Y, SHLADOVER S E, et al. A survey on cooperative longitudinal motion control of multiple connected and automated vehicles [J]. IEEE Intelligent Transportation Systems Magazine, 2020, 12(1): 4-24.

[4] RAJAMANI R, ZHU C. Semi-autonomous adaptive cruise control systems [J]. IEEE Transactions on Vehicular Technology, 2002, 51(5): 1186-1192.

[5] BAROOAH P, HESPANHA J P. Error amplification and disturbance propagation in vehicle strings with decentralized linear control $[\mathrm{C}] / /$ Proceedings of the 44 th IEEE Conference on Decision and Control. Seville: IEEE, 2005: 4964-4969.

[6] BESSELINK B, JOHANSSON K H. String stability and a delay-based spacing policy for vehicle platoons subject to disturbances [J]. IEEE Transactions on $\mathrm{Au}$ tomatic Control, 2017, 62(9): 4376-4391.

[7] NAUS G J L, VUGTS R P A, PLOEG J, et al. Stringstable CACC design and experimental validation: A frequency-domain approach $[\mathrm{J}]$. IEEE Transactions on Vehicular Technology, 2010, 59(9): 4268-4279.

[8] PLOEG J, SCHEEPERS B T M, VAN NUNEN E, et al. Design and experimental evaluation of cooperative adaptive cruise control $[\mathrm{C}] / / 2011$ 14th International IEEE Conference on Intelligent Transportation Systems (ITSC). Washington, DC: IEEE, 2011: 260265.

[9] GONG J, CAO J, ZHAO Y, et al. Sampling-based cooperative adaptive cruise control subject to communication delays and actuator lags [J]. Mathematics and Computers in Simulation, 2020, 171: 13-25.

[10] ZHANG Y, BAI Y, WANG M, et al. Cooperative adaptive cruise control with robustness against communication delay: An approach in the space domain $[\mathrm{J}]$. IEEE Transactions on Intelligent Transportation Systems, 2021, 22(9): 5496-5507.

[11] KWON J, CHWA D. Adaptive bidirectional platoon control using a coupled sliding mode control method [J]. IEEE Transactions on Intelligent Transportation Systems, 2014, 15(5): 2040-2048.

[12] WU Y, LI S E, ZHENG Y, et al. Distributed sliding mode control for multi-vehicle systems with positive definite topologies [C]//2016 IEEE 55th Conference on Decision and Control $(C D C)$. Las Vegas, NV: IEEE, 2016: 5213-5219.
[13] YAN M, SONG J, YANG P, et al. Distributed adaptive sliding mode control for vehicle platoon with uncertain driving resistance $[\mathrm{C}] / / 2017$ 36th Chinese Control Conference $(C C C)$. Dalian: IEEE, 2017: 9396-9400.

[14] GUO G, LI D. Adaptive sliding mode control of vehicular platoons with prescribed tracking performance [J]. IEEE Transactions on Vehicular Technology, 2019, 68(8): 7511-7520.

[15] AGHABABA M P, SAIF M. Finite time sliding mode control of connected vehicle platoons guaranteeing string stability $[\mathrm{C}] / / 2020$ IEEE International Conference on Human-Machine Systems (ICHMS). Rome: IEEE, 2020: 1-6.

[16] KIANFAR R, FALCONE P, FREDRIKSSON J. A receding horizon approach to string stable cooperative adaptive cruise control [C]//2011 14th International IEEE Conference on Intelligent Transportation Systems (ITSC). Washington, DC: IEEE, 2011: 734-739.

[17] ZHENG Y, LI S E, LI K, et al. Distributed model predictive control for heterogeneous vehicle platoons under unidirectional topologies [J]. IEEE Transactions on Control Systems Technology, 2017, 25(3): 899-910.

[18] PLOEG J, SHUKLA D P, VAN DE WOUW N, et al. Controller synthesis for string stability of vehicle platoons [J]. IEEE Transactions on Intelligent Transportation Systems, 2014, 15(2): 854-865.

[19] PLOEG J, VAN DE WOUW N, NIJMEIJER H. Fault tolerance of cooperative vehicle platoons subject to communication delay [J]. IFAC-PapersOnLine, 2015, 48(12): 352-357.

[20] GAO F, LI S E, ZHENG Y, et al. Robust control of heterogeneous vehicular platoon with uncertain dynamics and communication delay [J]. IET Intelligent Transport Systems, 2016, 10(7): 503-513.

[21] DESJARDINS C, CHAIB-DRAA B. Cooperative adaptive cruise control: A reinforcement learning approach [J]. IEEE Transactions on Intelligent Transportation Systems, 2011, 12(4): 1248-1260.

[22] GUO G, LI D. PMP-based set-point optimization and sliding-mode control of vehicular platoons [J]. IEEE Transactions on Computational Social Systems, 2018, 5(2): 553-562.

[23] FESHARAKI S J, KAMALI M, SHEIKHOLESLAM $\mathrm{F}$, et al. Robust model predictive control with sliding mode for constrained non-linear systems [J]. IET Control Theory \& Applications, 2020, 14(17): 2592-2599.

[24] GUO X, WANG J, LIAO F, et al. CNN-based distributed adaptive control for vehicle-following platoon with input saturation [J]. IEEE Transactions on Intelligent Transportation Systems, 2018, 19(10): 31213132 .

[25] FENG Y, YU X, MAN Z. Non-singular terminal sliding mode control of rigid manipulators [J]. Automatica, 2002, 38(12): 2159-2167. 